Article

\title{
Cantharidin and Its Anhydride-Modified Derivatives: Relation of Structure to Insecticidal Activity
}

\section{Wenbo Sun, Zhongyi Liu and Yalin Zhang *}

Key Laboratory of Plant Protection Resources \& Pest Management of Ministry of Education, Northwest A \& F University, Yangling 712100, Shaanxi, China;

E-Mails: wbsun06@yahoo.com.cn (W.S.); zhy.liu@hotmail.com (Z.L.)

* Author to whom correspondence should be addressed; E-Mail: yalinzh@yahoo.com.cn; Tel./Fax: +86-29-8709-2190.

Received: 21 September 2012; in revised form: 12 November 2012 / Accepted: 26 November 2012 / Published: 20 December 2012

\begin{abstract}
Cantharidin is a natural compound of novel structure with ideal insecticidal activity. However, the relationship of structure to insecticidal activity of cantharidin and its derivatives has not been ever clarified. To explore what determines the insecticidal activity structurally of cantharidin-related compounds, two series target compounds $\mathbf{6}$ and 7 were synthesized by replacing the anhydride ring of norcantharidin with an aromatic amine or fatty amine with different electron density, respectively. The structures of these compounds were characterized by ${ }^{1} \mathrm{H}$ NMR, ${ }^{13} \mathrm{C}$ NMR and HRMS-ESI. A bioassay showed that compounds $\mathbf{6}(\mathbf{a}-\mathbf{m})$ lacked any larvicidal activity against Plutella xylostella; whereas their ring-opened partners $7(\mathbf{a}-\mathbf{m})$ provided a variety of larvicidal activities against P. xylostella, and compound $\mathbf{7 f}$ indicated the highest larvicidal activity with $\mathrm{LC}_{50}$ value of $0.43 \mathrm{mM}$. The present work demonstrated that the form of the compound (cyclic or ring-opened) or their ability to hydrolyze facilely was the key to determine whether it exhibits larvicidal activity. Moreover, it revealed that the improvement of insecticidal activity required a reasonable combination of both aliphatic amide and aromatic amide moieties, and the type of substituent $\mathrm{Y}$ on the aniline ring was critical.
\end{abstract}

Keywords: cantharidin; anhydride ring opening; derivatives; synthesis; larvicidal activity; structure-activity relationship 


\section{Introduction}

The blister beetle Mylabris phalerata (Coleoptera: Meloidae) has been used for centuries as a traditional Chinese medicine. Cantharidin 1 (Figure 1), the active ingredient in the blister beetle toxin, has served as a valuable drug against several kinds of cancer. Similar to what occurs when a variety of human cancer cell lines are co-cultured with cantharidin, insect cell lines Spex-VII and Sf9 can be induced to undergo apoptosis by this toxin [1-4]. Among the novel applications of cantharidin and its analogs were preliminary studies of the anti-insect activity of this natural product.

Figure 1. The structures of cantharidin 1 and norcantharidin 3.

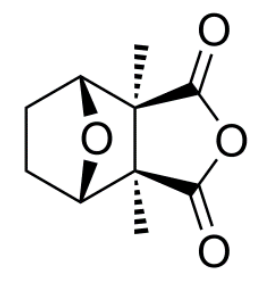

Cantharidin 1

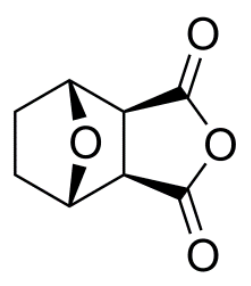

Norcantharidin $\mathbf{3}$

Cantharidin has been confirmed to be toxic to arthropods in a diversity of orders: Coleoptera, Diptera, Hymenoptera, Homoptera, Lepidoptera and Acarina [5-7]. Apoptosis-like symptoms of insect tissues (midgut, Malpighian tubules, testes, fat body and epidermis) due to cantharidin poisoning of insects were observed under the transmission electron microscope [8-10]. Additionally, the activities of acid phosphatase, alkaline phosphatase and polyphenol oxidase in cantharidin-treated Mythimna separata significantly declined [11].

Whereas the procedure for synthesizing cantharidin under assay conditions is complex and difficult [12-14], its analogs are much easier to be synthesized and are promising substitutes for cantharidin. However, cantharidin derivatives possess different structures and are likely to have either higher or lower insecticidal effects.

Our study is designed to explore the relationships between the structures and insecticidal activities of cantharidin analogs. Two series of anhydride-modified cantharidin analogs were designed and synthesized with norcantharidin 3 (Figure 1) as the parent compound. Among them, 6b, 6g, 6m, 7a, 7b, $7 \mathrm{e}$ and $7 \mathrm{f}$, seven derivatives, were new compounds. Their insecticidal activities were evaluated against the pre-third-instar larvae of Plutella xylostella, in vivo.

\section{Results and Discussion}

\subsection{Chemistry}

As shown in Scheme 1, the target analogs 6 were synthesized by the reaction of norcantharidin 3 and various primary amines in the presence of triethylamine as the binding acid agent [15], and the analogues 7 were synthesized by replacing the anhydride ring of norcantharidin 3 with an aromatic amine or fatty amine of different electron density [16].

Compound 5 was synthesized to imitate the synthetic procedure of phthalimide [17], as shown in Scheme 2 . 
Scheme 1. The structure and synthesis of compounds 6 and 7.<smiles></smiles>

3
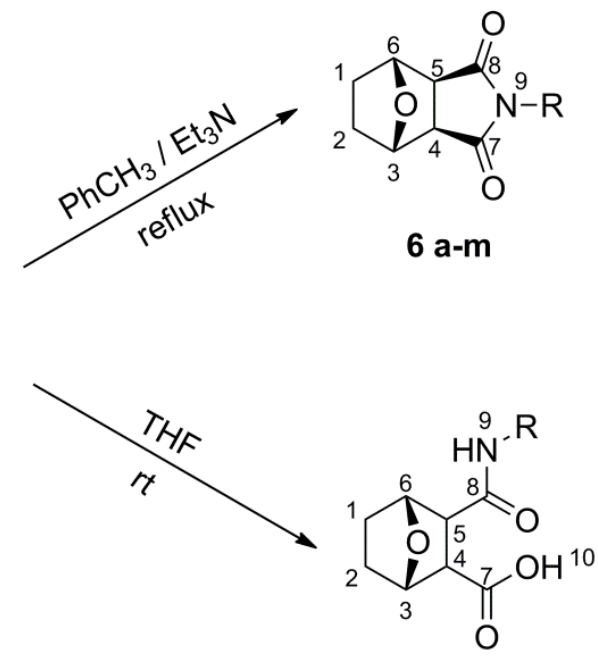

7 a-m

$\mathrm{R}=$

$\mathrm{H}_{3} \mathrm{C}-\xi$

a<smiles>COc1ccccc1</smiles>

d<smiles>C[As]1CCCC1</smiles>

b

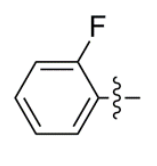

e

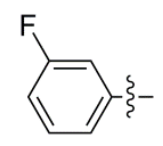

h<smiles>CCCC(C)(C)C</smiles>

C<smiles>O=[N+]([O-])c1ccccc1F</smiles><smiles>Fc1cccc(C(F)(F)F)c1</smiles>

i<smiles>COc1ccc(F)cc1</smiles>

j<smiles>COc1cccc(F)c1</smiles>

g

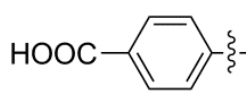

k

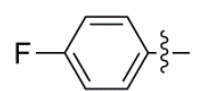

I

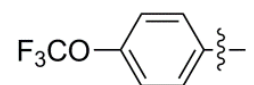

m

Scheme 2. The structure and synthesis of compound $\mathbf{5}$.<smiles>O=C1OC(=O)C2C3CCC(O3)C12</smiles>

3<smiles>NC(N)=O</smiles>
$\underset{160^{\circ} \mathrm{C}}{\stackrel{\mathrm{DMF}}{\longrightarrow}}$

As can be seen from Schemes 3 and 4, compounds $\mathbf{1}$ and $\mathbf{3}$ underwent a rapid conversion to dicarboxylic acid (compounds $\mathbf{2}$ and $\mathbf{4}$ ) under assay conditions, or through the slow but facile way that the hydrolyzing occurred at room temperature.

Thus the hydrolytic stabilities of analogs 6 presented in Table 1 were examined. We noted that analogs 6 were stable under assay conditions, showing no decomposition even under infrared radiation (typically no decomposition after $12 \mathrm{~h}$ treatment). 
Scheme 3. The structure and synthesis of compound 2.

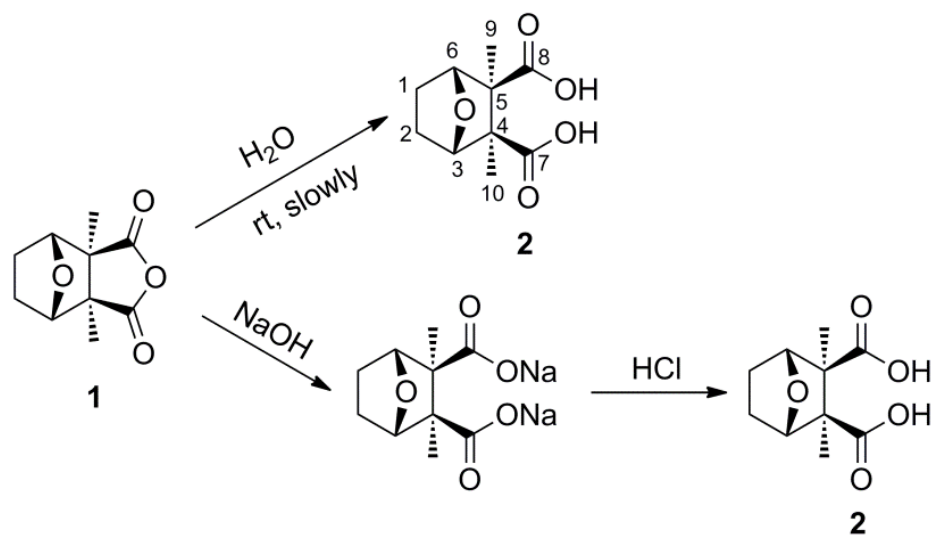

Scheme 4. The structure and synthesis of compound 4.

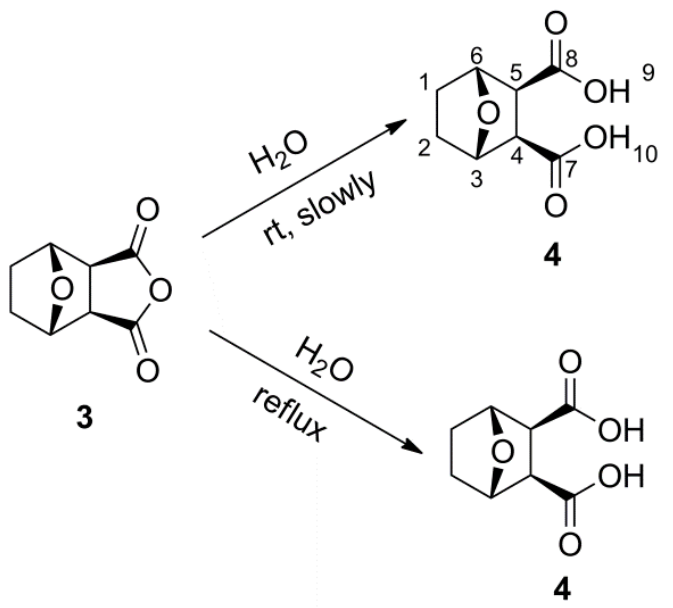

Table 1. Structures and larvicidal activities against $P$. xylostella of compounds 5, 6, 1 and $\mathbf{3 .}$

\begin{tabular}{|c|c|c|c|}
\hline Compounds & $\mathbf{R}$ & Concentration $\left(\mu \mathrm{g} \mathrm{mL}^{-1}\right)$ & Mortality (\%) \\
\hline 5 & $\mathrm{H}$ & 500 & 0 \\
\hline $6 a$ & $-\mathrm{CH}_{3}$ & 500 & 0 \\
\hline $6 b$ & $-\mathrm{CH}\left(\mathrm{CH}_{3}\right)_{2}$ & 500 & 0 \\
\hline $6 c$ & $-\mathrm{CH}_{2}\left(\mathrm{CH}_{2}\right)_{2} \mathrm{CH}_{3}$ & 500 & 0 \\
\hline $6 \mathrm{~d}$ & 2'-OMePh & 500 & 0 \\
\hline $6 e$ & 2'-FPh & 500 & 0 \\
\hline $6 f$ & $2^{\prime}-\mathrm{NO}_{2} \mathrm{Ph}$ & 500 & 0 \\
\hline $6 \mathrm{~g}$ & 3'-OMePh & 500 & 0 \\
\hline $6 \mathrm{~h}$ & 3'-FPh & 500 & 0 \\
\hline $6 \mathrm{i}$ & $3^{\prime}-\mathrm{CF}_{3} \mathrm{Ph}$ & 500 & 0 \\
\hline $6 \mathrm{j}$ & 4'-OMePh & 500 & 0 \\
\hline $6 \mathrm{k}$ & 4'- $\mathrm{CO}_{2} \mathrm{HPh}$ & 500 & 0 \\
\hline 61 & 4'-FPh & 500 & 0 \\
\hline $6 \mathrm{~m}$ & $4^{\prime}-\mathrm{OCF}_{3} \mathrm{Ph}$ & 500 & 0 \\
\hline Cantharidin 1 & - & 500 & 100 \\
\hline Norcantharidin 3 & - & 500 & 100 \\
\hline
\end{tabular}




\subsection{Insecticidal Activity}

After a period of starvation, released larvae wriggled around and attempted to eat the nearby treated leaves. They would not present a series of symptoms until a certain amount of the drug was taken. All active compounds caused the same symptoms.

Unlike most commercial pesticides currently used, the poisoned larvae gradually lost their locomotive ability and were powerless to resist mechanical irritations. Meanwhile, compared with the healthy larvae (Figure 2A,B), a darker patch often appeared anteriorly on each dying larva (Figure 2C), which would spread gradually to the whole bodies until they died (Figure 2D). Wet, green frass might stick to the anal areas of dying larvae (Figure 2D), and mucus was frequently observed at the end of the abdomen of dead larvae, which either was kept between the fourth pair of prolegs and caudal prolegs (Figure 2E), or it glued dying larvae to leaves or filter papers (Figure 2F) indicating the experimental drugs might disrupt the digestive system in larvae.

Figure 2. (A, B) Healthy larvae with light and uniform body color; (C) A darker patch appeared anteriorly on a dying larva poisoned by cantharidin; (D) Darker patches spread all over the body of a dead larva poisoned by cantharidin with wet, green frass stuck to its anal area, as shown at the arrow; (E) Mucus was kept between the fourth pair of prolegs and caudal prolegs as shown at the arrow; (F) A larva that died from cantharidin was glued posteriorly and ventrally to a leaf by mucus.

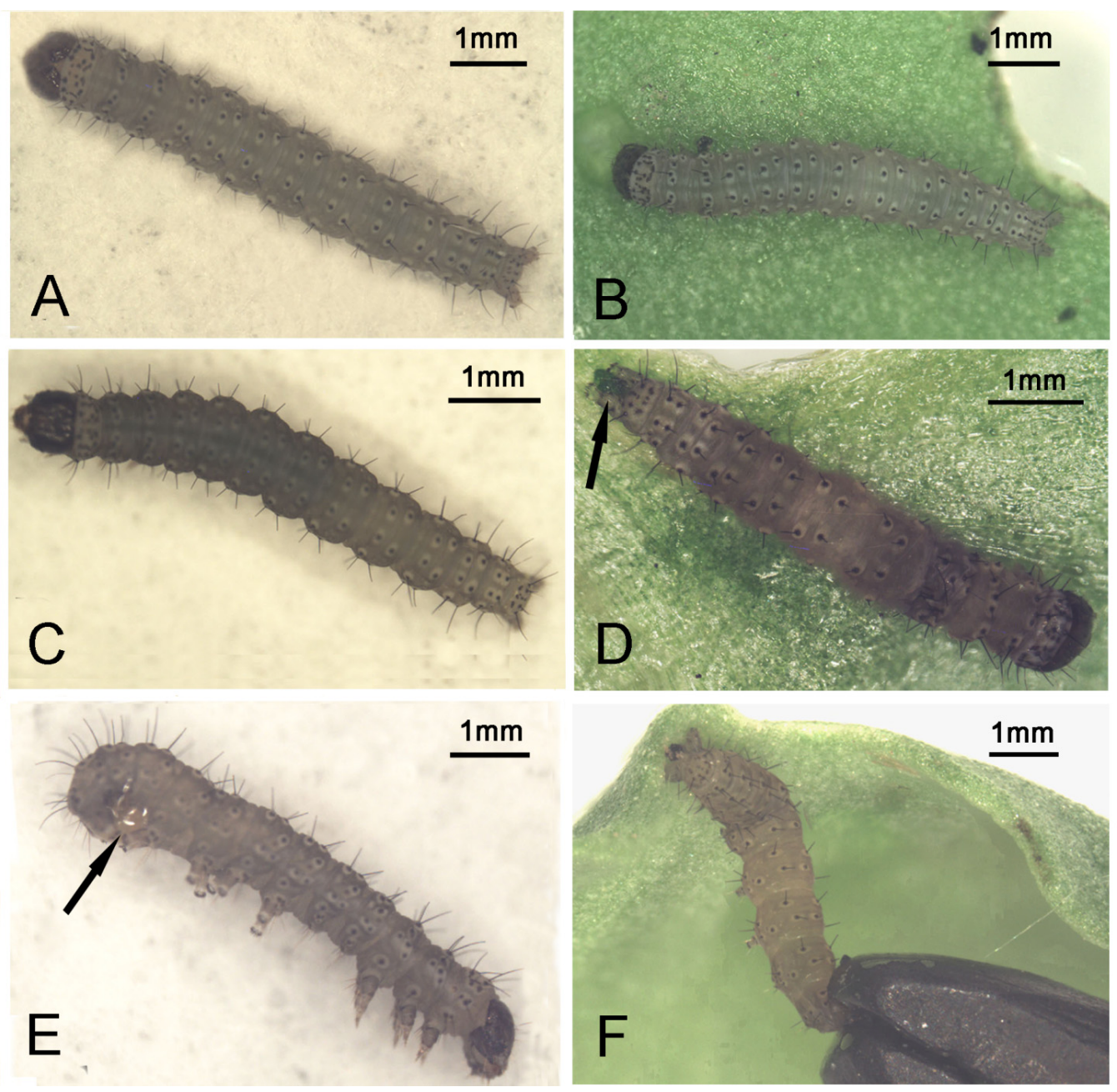


In addition, cantharidin is well known as a strong inhibitor of serine/threonine protein phosphatases (PPs) [18], a broad class of PPs associated with signaling and control of numerous cellular processes in many organisms [19]. The toxicity associated with cantharidin is derived from its ability to inhibit the family of PPs. However, the catalytic domain of all PP subfamilies is highly conserved in animals, plants, protozoans and all eukaryotes [20]. Inhibitors, such as cantharidin and norcantharidin, bind to a hydrophobic pocket of the PP active site [21]. The structural similarity between cantharidin and norcantharidin has been apparent to animal scientists and similar mechanisms of action on animal serine/threonine PPs have been confirmed [22]. Like all other eukaryotes, the family of serine/threonine protein phosphatases belonging to insects and related arthropods should be considered since it is highly similar to that of mammals. Hence, it is deduced that PPs are the potential target sites of insecticidal activity of cantharidin and its related compounds. Meanwhile, because of the high conservation of the catalytic domain of all PP subfamilies, cantharidin and its derivatives are indeed toxic to all eukaryotes, not just insects.

\section{3. $S A R$}

Among the sixteen compounds listed in Table 1, only cantharidin $\mathbf{1}$ and norcantharidin $\mathbf{3}$ showed any significant larvicidal activities with $100 \%$ mortality at concentrations of $500 \mu \mathrm{g} \mathrm{mL}^{-1}$, respectively. Interestingly, the replacement of the anhydride oxygen atom of norcantharidin 3 with nitrogen (compound 5) resulted in complete loss of activity. Meanwhile, Table 1 showed that the target compounds $\mathbf{6}$ also lacked any larvicidal activities. Indeed, to result in any larvicidal activity, no modifications of the cyclic anhydride are tolerated, consequently resulting in larvicidal activity. We thought the replacement of the $\mathrm{O}$-atom with $\mathrm{N}$ (as $\mathrm{N}-\mathrm{H}$ and $\mathrm{N}-\mathrm{R}$, where $\mathrm{R}=$ alkyl of aryl) would furnish us with a better understanding of the relationship between the electronic effect and larvicidal activities; however no larvicidal activity was demonstrated for the modified cyclic anhydride.

The biological activities of the target compounds $\mathbf{7 a}$ to $\mathbf{7 m}$ against $P$. xylostella at a concentration of $500 \mu \mathrm{g} \mathrm{mL}^{-1}$ are summarized in Table 2 . To provide structure-activity relationship information about the effect of the aliphatic amide moiety substituent, $\mathrm{R}$, compounds $\mathbf{7 a}, \mathbf{7 b}$ and $\mathbf{7 c}$ were designed to contain $-\mathrm{CH}_{3},-\mathrm{CH}\left(\mathrm{CH}_{3}\right)_{2}$ and $-\mathrm{CH}_{2}\left(\mathrm{CH}_{2}\right)_{2} \mathrm{CH}_{3}$, respectively. Although it is difficult to construct a clear structure-activity relationship from the data shown in Table 2, it can be concluded that the general trend in larvicidal activity for the substituents was $-\mathrm{CH}_{3}(\mathbf{7 a})>-\mathrm{CH}\left(\mathrm{CH}_{3}\right)_{2}(\mathbf{7 b})>-\mathrm{CH}_{2}\left(\mathrm{CH}_{2}\right)_{2} \mathrm{CH}_{3}$ (7c). For example, compound $7 \mathbf{a}\left(\mathrm{R}=-\mathrm{CH}_{3}, 77 \%\right)$ displayed a significantly higher insecticidal activity than compound $7 \mathbf{b}\left(\mathrm{R}=-\mathrm{CH}\left(\mathrm{CH}_{3}\right)_{2}, 12 \%\right)$ and $7 \mathbf{c}\left(\mathrm{R}=-\mathrm{CH}_{2}\left(\mathrm{CH}_{2}\right)_{2} \mathrm{CH}_{3}, 4 \%\right)$ at $500 \mu \mathrm{g} \mathrm{mL}{ }^{-1}$.

To examine the electronic effect of substituent $\mathrm{Y}$ on the aniline ring, the electron-donating substituent $-\mathrm{OCH}_{3}$ and electron-withdrawing substituents $-\mathrm{CF}_{3},-\mathrm{OCF}_{3}, \mathrm{~F}$ and $-\mathrm{CO}_{2} \mathrm{H}$ were introduced. Compounds with electron-withdrawing substituents displayed higher larvicidal activities against $P$. xylostella than compounds with electron-donating substituents, as seen in the comparison of the compounds $\mathbf{7 d}\left(\mathrm{Y}=2^{\prime}-\mathrm{OCH}_{3}\right), \mathbf{7 e}\left(\mathrm{Y}=2^{\prime}-\mathrm{F}\right)$ and $\mathbf{7 f}\left(\mathrm{Y}=2^{\prime}-\mathrm{NO}_{2}\right)$ of the series with $\mathrm{Y}$ at 2'-position on the aniline ring, $7 \mathbf{g}\left(\mathrm{Y}=3^{\prime}-\mathrm{OCH}_{3}\right)$, $7 \mathbf{h}\left(\mathrm{Y}=3^{\prime}-\mathrm{F}\right)$ and $7 \mathbf{i}\left(\mathrm{Y}=3^{\prime}-\mathrm{CF}_{3}\right)$ of the series with $\mathrm{Y}$ at 3'-position on the aniline ring, and $\mathbf{7 j}\left(\mathrm{Y}=4^{\prime}-\mathrm{OCH}_{3}\right), \mathbf{7 k}\left(\mathrm{Y}=4^{\prime}-\mathrm{CO}_{2} \mathrm{H}\right), 7 \mathbf{l}\left(\mathrm{Y}=4^{\prime}-\mathrm{F}\right)$ and $\mathbf{7 m}$ $\left(\mathrm{Y}=4^{\prime}-\mathrm{OCF}_{3}\right)$ of the series with $\mathrm{Y}$ at 4 '-position on the aniline ring. These observations revealed that substitution patterns on the aniline ring have an important influence on the larvicidal activity. 
Compounds with electron-withdrawing substituents showed excellent larvicidal activities against P. xylostella, while compounds with electron-donating substituents display lower larvicidal activity.

Table 2. Structures and larvicidal activities against $P$. xylostella of compounds 7, 2 and 4.

\begin{tabular}{|c|c|c|c|}
\hline Compounds & $\mathbf{R}$ & Concentration $\left(\mu \mathrm{g} \mathrm{mL}^{-1}\right)$ & Mortality (\%) \\
\hline $7 \mathrm{a}$ & $-\mathrm{CH}_{3}$ & 500 & 77 \\
\hline $7 b$ & $-\mathrm{CH}\left(\mathrm{CH}_{3}\right)_{2}$ & 500 & 12 \\
\hline $7 \mathrm{c}$ & $-\mathrm{CH}_{2}\left(\mathrm{CH}_{2}\right)_{2} \mathrm{CH}_{3}$ & 500 & 4 \\
\hline $7 d$ & 2'-OMePh & 500 & 25 \\
\hline $7 \mathrm{e}$ & 2'-FPh & 500 & 60 \\
\hline $7 f$ & $2^{\prime}-\mathrm{NO}_{2} \mathrm{Ph}$ & 500 & 100 \\
\hline $7 \mathrm{~g}$ & 3'-OMePh & 500 & 24 \\
\hline $7 \mathrm{~h}$ & 3'-FPh & 500 & 41 \\
\hline $7 \mathrm{i}$ & 3'- $\mathrm{CF}_{3} \mathrm{Ph}$ & 500 & 32 \\
\hline $7 \mathrm{j}$ & 4'-OMePh & 500 & 11 \\
\hline $7 \mathrm{k}$ & 4'- $\mathrm{CO}_{2} \mathrm{HPh}$ & 500 & 97 \\
\hline 71 & 4'-FPh & 500 & 38 \\
\hline $7 \mathrm{~m}$ & $4^{\prime}-\mathrm{OCF}_{3} \mathrm{Ph}$ & 500 & 19 \\
\hline 2 & - & 500 & 100 \\
\hline 4 & - & 500 & 100 \\
\hline
\end{tabular}

In addition, as shown in Table 2, compound 2, 4 and $\mathbf{7 f}$ were the most active compounds. All of their larvicidal activities against $P$. xylostella at $500 \mu \mathrm{g} \mathrm{mL} \mathrm{m}^{-1}$ were $100 \%$ after $48 \mathrm{~h}$, while the larvicidal activity of the parent compounds $\mathbf{1}$ and $\mathbf{2}$ were $100 \%$ at the same concentration after $48 \mathrm{~h}$ as shown in Table 1. These results indicated that compounds 2, $\mathbf{4}$ and $\mathbf{7 f}$ displayed comparable larvicidal activity with their corresponding lead compound against $P$. xylostella at $500 \mu \mathrm{g} \mathrm{mL}^{-1}$. Therefore, we carried out further insecticidal activity assay for compounds 2, 4 and 7f, and cantharidin $\mathbf{1}$ and norcantharidin 2 were used as a control to make a judgment on the larvicidal potency of these compounds. As shown in Table 3, it was found that the $\mathrm{LC}_{50}$ values of compounds 4 and $7 \mathbf{f}$ against P. xylostella were $0.70 \mathrm{mM}$ and $0.43 \mathrm{mM}$ respectively, while that of the parent compound norcantharidin was $0.84 \mathrm{mM}$. Moreover, the $\mathrm{LC}_{50}$ of compounds 2 was the same as that of its parent compound cantharidin 1 with value of $0.06 \mathrm{mM}$.

Table 3. Insecticidal activities against $P$. xylostella of compounds $\mathbf{1}-\mathbf{4}$ and $\mathbf{7 f}$.

\begin{tabular}{ccccc}
\hline Compounds & $\boldsymbol{y}=\mathbf{a}+\mathbf{b} \boldsymbol{x}$ & $\boldsymbol{r}$ & $\left.\mathbf{L C}_{\mathbf{5 0}} \mathbf{( 9 5 \%} \mathbf{C I}\right) \mathbf{( m M )}$ & $\mathbf{L C}_{\mathbf{9 0}}(\mathbf{9 5 \%} \mathbf{C I})(\mathbf{m M})$ \\
\hline Cantharidin 1 & $y=9.4287+3.6777 x$ & 0.99 & $0.06(0.06-0.07)$ & $0.14(0.11-0.17)$ \\
2 & $y=9.6322+3.8866 x$ & 0.99 & $0.06(0.05-0.07)$ & $0.14(0.11-0.18)$ \\
Norcantharidin 3 & $y=5.4052+5.1887 x$ & 0.97 & $0.84(0.75-0.93)$ & $1.48(0.94-1.97)$ \\
4 & $y=5.8547+5.4057 x$ & 0.98 & $0.70(0.64-0.77)$ & $1.20(0.94-1.52)$ \\
$7 \mathrm{f}$ & $y=6.8918+5.2902 x$ & 0.99 & $0.43(0.39-0.48)$ & $0.76(0.59-0.99)$ \\
\hline
\end{tabular}

The larvicidal activity values of anhydride analogs in Table 1 were compared with those of their ring-opened dicarboxylic acid partners in Table 2. The anhydride analogs indicated no any larvicidal activity, except $\mathbf{1}$ and $\mathbf{3}$, as mentioned above. However, their ring-opened dicarboxylic acid partners all 
suggested various larvicidal activities. On the one hand, the larvicidal activities of cantharidin $\mathbf{1}$ and norcantharidin 3 were equal to that of their ring-opened partners, compounds 2 and $\mathbf{4}$ with $\mathrm{LC}_{50}$ values of $0.06 \mathrm{mM}, 0.84 \mathrm{mM}, 0.06 \mathrm{mM}$, and $0.70 \mathrm{mM}$ in Table 3, respectively. And the larvicidal activities of cantharidin 1 and norcantharidin $\mathbf{3}$ appeared to be the results of the spontaneous hydrolyzing process to ring-opened compounds 2 and 4 . On the other hand, compared with 1 and 3, compounds 6 were so stable that they could not be hydrolyzed to their ring-opened partners naturally, which leads to the absence of larvicidal activity. Therefore, we conjectured that the active form of cantharidin and its derivatives is the dicarboxylic acid analog, a ring-opened compound.

\section{Experimental Section}

\subsection{General Experimental Procedures}

Melting points of all compounds were determined on a WRS-2 apparatus (Shanghai Precision \& Scientific Instrument Co. Ltd., Shanghai, China) and are uncorrected. ${ }^{1} \mathrm{H}$ NMR and ${ }^{13} \mathrm{C}$ NMR spectra were obtained using a Bruker AVANCE-500 $\mathrm{MHz}$ spectrometer in $\mathrm{CDCl}_{3}$ or DMSO solution with tetramethylsilane as the internal standard. Chemical shift values $(\delta)$ were given in parts per million (ppm). High-resolution mass spectrometry (HRMS) data were obtained on a Bruker micrOTOF-Q II instrument.

Cantharidin 1 was isolated from Mylabris phalerata (Chinese blister beetle), which was bought from the Chinese herbal medicine market in Xian, China. Norcantharidin 3 was purchased from Alfa Aesar Chemical Co. Ltd. (Haverhill, MA, USA). All primary amine reagents were of analytical reagent grade. Solvents were dried and purified using standard techniques immediately before use. Silica gel for TLC and CC was obtained from Qingdao Haiyang Chemical Co. Ltd. (Qingdao, China).

\subsection{Synthetic Procedures}

\subsubsection{General Synthetic Procedure for Compound 2}

To a solution of cantharidin $1(390 \mathrm{mg}, 2 \mathrm{mmol})$ in water $(10 \mathrm{~mL})$ was dropwise added a solution of sodium hydroxide $(180 \mathrm{mg}, 4.5 \mathrm{mmol})$ in water $(5 \mathrm{~mL})$. This was stirred at $100{ }^{\circ} \mathrm{C}$ for $2 \mathrm{~h}$. After the mixture had cooled to room temperature, a solution of hydrogen chloride $(4.5 \mathrm{mmol})$ in water $(9 \mathrm{~mL})$ was added dropwise to the mixture and stirred overnight at room temperature. The white slurry was filtered and the cake washed with dichloromethane. The crude solid was recrystallized from methanol to give 2 as white solid (400 mg, 93\%). mp: 232-233 ${ }^{\circ} \mathrm{C} .{ }^{1} \mathrm{H}$ NMR (500 MHz, D $\left.2 \mathrm{O}\right) \delta: 1.03(\mathrm{~s}, 6 \mathrm{H}$, H-9, 10), 1.44 (d, $J=8.51 \mathrm{~Hz}, 2 \mathrm{H}, \mathrm{H}-1,2$, exo), 1.76 (d, $J=7.88 \mathrm{~Hz}, 2 \mathrm{H}, \mathrm{H}-1,2$, endo), 4.53 (br, s, 2H, H-3, 6). ${ }^{13} \mathrm{C}$ NMR (125 MHz, $\left.\mathrm{D}_{2} \mathrm{O}\right) \delta: 17.26$ (C-9, C-10), 23.98 (C-1, C-2), 58.45 (C-4, C-5), 84.29 (C-3, C-6), 183.94 (C-7, C-8). HR-MS (ESI): $m / z$ calcd for $\mathrm{C}_{10} \mathrm{H}_{13} \mathrm{O}_{5}\left([\mathrm{M}-1]^{-}\right)$213.0763, found 213.0773 .

\subsubsection{General Synthetic Procedure for Compound 4}

A mixture of norcantharidin $3(1.0 \mathrm{~g}, 5.95 \mathrm{mmol})$ and water $(15 \mathrm{~mL})$ was refluxed for $8 \mathrm{~h}$. After the mixture had cooled to room temperature, the reaction was concentrated in vacuo and filtered. The 
resulting precipitate was recrystallized from methanol to obtain compound 4 as white solid (960 $\mathrm{mg}$, 87\%). mp: $137-138{ }^{\circ} \mathrm{C} .{ }^{1} \mathrm{H}$ NMR (500 MHz, DMSO-d 6 ) $\delta: 1.48-1.60$ (m, 4H, H-1, 2), 2.92 (s, 2H, $\mathrm{H}-4,5), 4.67$ (d, $J=2.21 \mathrm{~Hz}, 2 \mathrm{H}, \mathrm{H}-3,6), 12.05$ (br, s, 2H, H-9, 10). ${ }^{13} \mathrm{C}$ NMR (125 MHz, DMSO-d 6 )

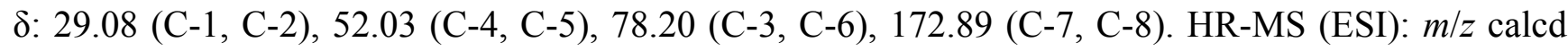
for $\mathrm{C}_{8} \mathrm{H}_{9} \mathrm{O}_{5}\left([\mathrm{M}-1]^{-}\right) 185.0450$, found 185.0455 .

\subsubsection{General Synthetic Procedure for Compound 5}

A mixture of norcantharidin $3(1.0 \mathrm{~g}, 5.95 \mathrm{mmol})$, carbamide $(220 \mathrm{mg}, 3.57 \mathrm{mmol})$ and DMF $(1.4 \mathrm{~mL})$ was heated at $130-135^{\circ} \mathrm{C}$. After the mixture had melted completely, it was allowed to react at $160-165^{\circ} \mathrm{C}$. When the reaction was completed according to TLC analysis, the mixture cooled to room temperature. After filtration, the crude solid was recrystallized from EtOAc/Hexane $(1: 1, v / v)$ resulting in product 5 as yellow solid $(920 \mathrm{mg}, 88.5 \%)$. mp: $186-188{ }^{\circ} \mathrm{C} .{ }^{1} \mathrm{H}$ NMR $\left(500 \mathrm{MHz}, \mathrm{CDCl}_{3}\right)$ $\delta: 1.60(\mathrm{~d}, J=7.57 \mathrm{~Hz}, 2 \mathrm{H}, \mathrm{H}-1,2$, exo), 1.87 (d, $J=7.57 \mathrm{~Hz}, 2 \mathrm{H}, \mathrm{H}-1,2$, endo), 2.93 (br, s, 2H, H-4, 5), 4.92 (br, s, 2H, H-3, 6), 8.33 (br, s, 1H, H-9). ${ }^{13} \mathrm{C} \mathrm{NMR} \mathrm{(125} \mathrm{MHz,} \mathrm{CDCl}_{3}$ ) $\delta: 28.52$ (C-1, C-2), 51.36 (C-4, C-5), 79.12 (C-3, C-6), 177.23 (C-7, C-8). HR-MS (ESI): $m / z$ calcd for $\mathrm{C}_{8} \mathrm{H}_{9} \mathrm{NO}_{3} \mathrm{Na}\left([\mathrm{M}+\mathrm{Na}]^{+}\right)$ 190.0480 , found 190.0474 .

\subsubsection{General Synthetic Procedure for the Target Compounds $6(\mathbf{a}-\mathbf{m})$}

Primary amine ( 1 equiv, $5.59 \mathrm{mmol}$ ) was added to a stirred mixture of norcantharidin $\mathbf{3}(1.0 \mathrm{~g}$, $5.95 \mathrm{mmol})$, triethylamine $(1.5 \mathrm{~mL})$ and toluene $(15 \mathrm{~mL})$. The reaction was carried out at reflux temperature, and the progress was monitored by TLC. After completion, the mixture was moved to vacuo. The residue was either recrystallized from EtOAc/Hexane $(1: 1, v / v)$ or purified by column chromatography on a silica gel using EtOAc and hexane (EtOAc/Hexane, 2:5, v/v) as the eluent to afford the target compounds 6 . The yields, physical properties, ${ }^{1} \mathrm{H}$ NMR, ${ }^{13} \mathrm{C} \mathrm{NMR}$, and HR-MS of the target compounds 6 were as follows:

Data for 6-a: white solid; yield, 93\%; mp: $132-133{ }^{\circ} \mathrm{C} ;{ }^{1} \mathrm{H}$ NMR $\left(500 \mathrm{MHz}, \mathrm{CDCl}_{3}\right) \delta$ : $1.58-1.64$ ( $\mathrm{m}, 2 \mathrm{H}, \mathrm{H}-1,2$, exo), 1.83-1.90 (m, 2H, H-1, 2, endo), 2.89 (s, 2H, H-4, 5), 2.96 (s, 3H, H-1'), 4.88 (br, s, 2H, H-3, 6); ${ }^{13} \mathrm{C}$ NMR (125 MHz, $\mathrm{CDCl}_{3}$ ) $\delta: 25.07$ (C-1'), 28.59 (C-1, C-2), 50.03 (C-4, C-5), 79.00 (C-3, C-6), 177.26 (C-7, C-8); HR-MS (ESI): $m / z$ calcd for $\mathrm{C}_{9} \mathrm{H}_{11} \mathrm{NO}_{3} \mathrm{Na}\left([\mathrm{M}+\mathrm{Na}]^{+}\right) \quad 204.0637$, found 204.0633 .

Data for 6-b: white solid; yield, 71\%; mp: 141-142 ${ }^{\circ} \mathrm{C} ;{ }^{1} \mathrm{H}$ NMR (500 MHz, CDCl3) $\delta: 1.36$ (d, $\left.J=6.94 \mathrm{~Hz}, 6 \mathrm{H}, \mathrm{H}-2^{\prime}, 3^{\prime}\right), 1.56-1.62$ (m, 2H, H-1, 2, exo), 1.80-1.88 (m, 2H, H-1, 2, endo), 2.79 (s, 2H, H-4, 5), 4.32 (dt, $J=13.87,6.94 \mathrm{~Hz}, 1 \mathrm{H}, \mathrm{H}-1$ '), 4.82-4.89 (m, 2H, H-3, 6); 13C NMR (125 MHz, CDCl3) $\delta: 19.16$ (s, 2C, C-2', C-3'), 28.63 (s, 2C, C-1, C-2), 43.99 (s, 1C, C-1'), 49.58 (s, 2C, C-4, C-5), 79.18 (s, 2C, C-3, C-6), 177.27 (s, 2C, C-7, C-8); HR-MS (ESI): $m / z$ calcd for $\mathrm{C}_{11} \mathrm{H}_{15} \mathrm{NO}_{3} \mathrm{Na}\left([\mathrm{M}+\mathrm{Na}]^{+}\right) 232.0950$, found 232.0953 .

Data for 6-c: white solid; yield, 89\%; mp: 80-81 ${ }^{\circ} \mathrm{C} ;{ }^{1} \mathrm{H}$ NMR (500 $\left.\mathrm{MHz}, \mathrm{CDCl}_{3}\right) \delta: 0.91$ (t, $J=7.25 \mathrm{~Hz}, 3 \mathrm{H}, \mathrm{H}-4^{\prime}$ ), 1.29 (sxt, $J=7.31 \mathrm{~Hz}, 2 \mathrm{H}, \mathrm{H}-3$ '), 1.52 (quin, $J=7.41 \mathrm{~Hz}, 2 \mathrm{H}, \mathrm{H}-2^{\prime}$ ), 1.60 (d, $J=7.25 \mathrm{~Hz}, 2 \mathrm{H}, \mathrm{H}-1,2$, exo), 1.86 (d, $J=7.88 \mathrm{~Hz}, 2 \mathrm{H}, \mathrm{H}-1,2$, endo), 2.86 (s, 2H, H-4, 5), 3.46 $\left(\mathrm{t}, J=7.25 \mathrm{~Hz}, 2 \mathrm{H}, \mathrm{H}-1^{\prime}\right), 4.87$ (br, s, 2H, H-3, 6); ${ }^{13} \mathrm{C}$ NMR (125 MHz, $\left.\mathrm{CDCl}_{3}\right) \delta: 13.62\left(\mathrm{C}-44^{\prime}\right), 19.94$ 
(C-3'), 28.61 (C-1, C-2), 29.65 (C-2'), 38.88 (C-1'), 49.87 (C-4, C-5), 79.07 (C-3, C-6), 177.28 (C-7, C-8); HR-MS (ESI): $m / z$ calcd for $\mathrm{C}_{12} \mathrm{H}_{17} \mathrm{NO}_{3} \mathrm{Na}\left([\mathrm{M}+\mathrm{Na}]^{+}\right)$246.1106, found 246.1101.

Data for 6-d: white solid; yield, 71\%; mp: $149-150{ }^{\circ} \mathrm{C} ;{ }^{1} \mathrm{H}$ NMR (500 $\left.\mathrm{MHz}, \mathrm{CDCl}_{3}\right) \delta$ : 1.65 (d, $J=7.25 \mathrm{~Hz}, 2 \mathrm{H}, \mathrm{H}-1,2$, exo), 1.90 (d, $J=7.88 \mathrm{~Hz}, 2 \mathrm{H}, \mathrm{H}-1,2$, endo), 3.00-3.10 (m, 2H, H-4, 5), 3.76-3.82 (m, 3H, H-7'), 4.99 (d, $J=2.84$ Hz, 2H, H-3, 6), 6.97-7.05 (m, 2H, H-3', 4'), 7.06-7.17 (m, 1H, H-5'), 7.39 (t, $\left.J=7.72 \mathrm{~Hz}, 1 \mathrm{H}, \mathrm{H}-6{ }^{\prime}\right) ;{ }^{13} \mathrm{C} \mathrm{NMR}\left(125 \mathrm{MHz}, \mathrm{CDCl}_{3}\right) \delta: 28.66$ (C-1, C-2), 50.41 (C-4, C-5), 55.81 (C-7'), 79.43 (C-3, C-6), 112.09 (C-3'), 112.57 (C-6'), 120.98 (C-1'), 129.32 (C-5'), 130.84 (C-4'), 154.57 (C-2'), 176.28 (C-7, C-8); HR-MS (ESI): $m / z$ calcd for $\mathrm{C}_{15} \mathrm{H}_{15} \mathrm{NO}_{4} \mathrm{Na}$ $\left([\mathrm{M}+\mathrm{Na}]^{+}\right)$296.0899, found 296.1001 .

Data for 6-e: white solid; yield, 71\%; mp: $139-140{ }^{\circ} \mathrm{C} ;{ }^{1} \mathrm{H}$ NMR $\left(500 \mathrm{MHz}, \mathrm{CDCl}_{3}\right) \delta$ : $1.62-1.70$ (m, 2H, H-1, 2, exo), 1.87-1.95 (m, 2H, H-1, 2, endo), 3.09 (br, s, 2H, H-4, 5), 5.00 (br, s, 2H, H-3, 6), 7.18-7.22 (m, 1H, H-4'), 7.23 (br, s, 2H, H-3', 5'), 7.37-7.45 (m, 1H, H-6'); ${ }^{13} \mathrm{C}$ NMR (125 MHz, $\left.\mathrm{CDCl}_{3}\right) \delta: 28.64$ (s, 2C, C-1, C-2), 50.39 (s, 2C, C-4, C-5), 79.51 (s, 2C, C-3, C-6), 119.65 (s, 1C, C-3'), 124.67 (br, s, 1C, C-5'), 128.24 (s, 1C, C-1'), 129.42 (br. s, 1C, C-4'), 131.01 (s, 1C, C-6'), 156.29 (s, 1C, C-2'), 175.54 (s, 2C, C-7, C-8); HR-MS (ESI): $m / z$ calcd for $\mathrm{C}_{14} \mathrm{H}_{12} \mathrm{FNO}_{3} \mathrm{Na}\left([\mathrm{M}+\mathrm{Na}]^{+}\right)$ 284.0699, found 284.0691.

Data for 6-f: white solid; yield, 52\%; mp: $179-181{ }^{\circ} \mathrm{C} ;{ }^{1} \mathrm{H}$ NMR $\left(500 \mathrm{MHz}, \mathrm{CDCl}_{3}\right) \delta: 1.68$ (d, $J=7.25 \mathrm{~Hz}, 2 \mathrm{H}, \mathrm{H}-1,2$, exo), 1.92 (d, $J=7.88 \mathrm{~Hz}, 2 \mathrm{H}, \mathrm{H}-1,2$, endo), 3.11-3.21 (m, 2H, H-4, 5), 4.96-5.06 (m, 2H, H-3, 6), 7.41 (d, $J=7.57 \mathrm{~Hz}, 1 \mathrm{H}, \mathrm{H}-4$ '), 7.61 (t, $\left.J=7.57 \mathrm{~Hz}, 1 \mathrm{H}, \mathrm{H}-5^{\prime}\right), 7.70-7.80$ (m, 1H, H-6'), 8.18 (d, J=8.20 Hz, 1H, H-3'); ${ }^{13} \mathrm{C}$ NMR (125 MHz, $\mathrm{CDCl}_{3}$ ) $\delta: 28.61$ (C-1, C-2), 50.59 (C-4, C-5), 79.49 (C-3, C-6), 125.81 (C-6'), 130.19 (C-4'), 130.66 (C-3'), 134.39 (C-5'), 175.47 (C-7, C-8); HR-MS (ESI): $m / z$ calcd for $\mathrm{C}_{14} \mathrm{H}_{12} \mathrm{~N}_{2} \mathrm{O}_{5} \mathrm{Na}\left([\mathrm{M}+\mathrm{Na}]^{+}\right)$311.0644, found 311.0623 .

Data for 6-g: white solid; yield, 79\%; mp: $147-148{ }^{\circ} \mathrm{C} ;{ }^{1} \mathrm{H} \mathrm{NMR}\left(500 \mathrm{MHz}, \mathrm{CDCl}_{3}\right) \delta$ : $1.62-1.69$ (m, 2H, H-1, 2, exo), 1.87-1.95 (m, 2H, H-1, 2, endo), 3.03 (s, 2H, H-4, 5), 3.81 (s, 3H, H-7'), 5.00 (br, s, 2H, H-3, 6), 6.78 (s, 1H, H-4'), 6.84 (d, $\left.J=7.88 \mathrm{~Hz}, 1 \mathrm{H}, \mathrm{H}-2^{\prime}\right), 6.94$ (dd, $J=8.35,1.73 \mathrm{~Hz}, 1 \mathrm{H}$,

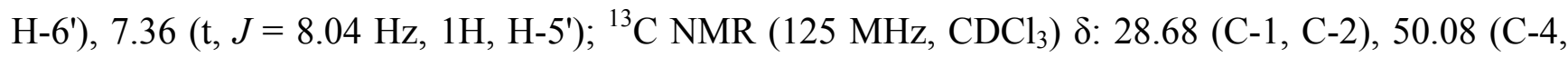
C-5), 55.48 (C-7'), 79.56 (C-3, C-6), 112.24 (C-2'), 114.91 (C-4'), 118.81 (C-6'), 129.89 (C-5'), 132.85 (C-1'), 160.14 (C-3'), 176.30 (C-7, C-8); HR-MS (ESI): $m / z$ calcd for $\mathrm{C}_{15} \mathrm{H}_{15} \mathrm{NO}_{4} \mathrm{Na}\left([\mathrm{M}+\mathrm{Na}]^{+}\right)$ 296.0899, found 296.1015.

Data for 6- $\boldsymbol{h}$ : white solid; yield, 85\%; mp: $139-140{ }^{\circ} \mathrm{C} ;{ }^{1} \mathrm{H}$ NMR $\left(500 \mathrm{MHz}, \mathrm{CDCl}_{3}\right) \delta$ : $1.64-1.70$ (m, 2H, H-1, 2, exo), 1.87-1.94 (m, 2H, H-1, 2, endo), 3.03 (s, 2H, H-4, 5), 4.99 (d, J=2.21 Hz, 2H, $\mathrm{H}-3,6), 7.11-7.18$ (m, 2H, H-4', 6'), 7.22-7.29 (m, 2H, H-2', 5'); $\left.{ }^{13} \mathrm{C} \mathrm{NMR} \mathrm{(125} \mathrm{MHz,} \mathrm{CDCl}_{3}\right) \delta: 28.66$ (s, 2C, C-1, C-2), 50.04 (s, 2C, C-4, C-5), 79.57 (s, 2C, C-3, C-6), 116.09 (s, 1C, C-2'), 116.28 (s, 1C, C-4'), 127.75 (s, 1C, C-6'), 128.35 (s, 1C, C-5'), 161.31 (s, 1C, C-1'), 163.29 (s, 1C, C-3'), 176.27 (s, 2C, C-7, C-8); HR-MS (ESI): $m / z$ calcd for $\mathrm{C}_{14} \mathrm{H}_{12} \mathrm{FNO}_{3} \mathrm{Na}\left([\mathrm{M}+\mathrm{Na}]^{+}\right)$284.0699, found 284.0692.

Data for 6-i: white solid; yield, 92\%; mp: 197-198 ${ }^{\circ} \mathrm{C} ;{ }^{1} \mathrm{H}$ NMR (500 MHz, $\left.\mathrm{CDCl}_{3}\right) \delta$ : $1.65-1.71$ (m, 2H, H-1, 2, exo), 1.89-1.96 (m, 2H, H-1, 2, endo), 3.06 (s, 2H, H-4, 5), 5.01 (d, J=2.21 Hz, 2H, H-3, 6), 7.50 (d, $\left.J=7.88 \mathrm{~Hz}, 1 \mathrm{H}, \mathrm{H}-6^{\prime}\right), 7.56-7.62$ (m, 2H, H-4', 5'), 7.63-7.68 (m, 1H, H-2'); ${ }^{13} \mathrm{C}$ NMR (125 MHz, $\mathrm{CDCl}_{3}$ ) $\delta: 28.65$ (s, 2C, C-1, C-2), 50.09 (s, 2C, C-4, C-5), 79.64 (s, 2C, C-3, C-6), 123.56 (s, 1C, C-4'), 124.59 (s, 1C, C-7'), 125.44 (s, 1C, C-2'), 129.70 (s, 1C, C-5'), 131.56 (s, 1C, C-3'), 131.82 (s, 1C, C-6'), 132.38 (s, 1C, C-1'), 175.88 (s, 2C, C-7, C-8); HR-MS (ESI): $m / z$ calcd for $\mathrm{C}_{15} \mathrm{H}_{12} \mathrm{~F}_{3} \mathrm{NO}_{3} \mathrm{Na}\left([\mathrm{M}+\mathrm{Na}]^{+}\right)$334.0667, found 334.0665 . 
Data for 6-j: white solid; yield, 85\%; mp: $189-190{ }^{\circ} \mathrm{C} ;{ }^{1} \mathrm{H}$ NMR $\left(500 \mathrm{MHz}, \mathrm{CDCl}_{3}\right) \delta$ : 1.66 (d, $J=7.25 \mathrm{~Hz}, 2 \mathrm{H}, \mathrm{H}-1,2$, exo), 1.90 (d, $J=8.20 \mathrm{~Hz}, 2 \mathrm{H}, \mathrm{H}-1,2$, endo), 3.02 (s, 2H, H-4, 5), 3.82 (s, 3H, H-7'), 4.99 (br, s, 2H, 2H, H-3, 6), 6.97 (d, J=8.83 Hz, 2H, H-3', 5'), 7.17 (d, J=8.83 Hz, 2H, $\left.\mathrm{H}-2^{\prime}, 6^{\prime}\right) ;{ }^{13} \mathrm{C}$ NMR (125 MHz, $\mathrm{CDCl}_{3}$ ) $\delta: 28.69$ (C-1, C-2), 40.48 (C-7'), 49.99 (C-4, C-5), 79.47 (C-3, C-6), 112.44 (C-3', C-5'), 120.25 (C-1'), 127.17 (C-2', C-6'), 150.57 (C-4'), 176.98 (C-7, C-8); HR-MS (ESI): $m / z$ calcd for $\mathrm{C}_{15} \mathrm{H}_{15} \mathrm{NO}_{4} \mathrm{Na}\left([\mathrm{M}+\mathrm{Na}]^{+}\right)$296.0899, found 296.1011 .

Data for 6-k: white solid; yield, \%; mp: 270-271 ${ }^{\circ} \mathrm{C}$; ${ }^{1} \mathrm{H}$ NMR (500 MHz, DMSO-d $\left.\mathrm{d}_{6}\right) \delta: 1.71$ (s, 4H, H-1, 2), 2.94 (q, J=7.25 Hz, 2H, H-4, 5), 4.83 (s, 2H, H-3, 6), 7.31 (d, J=8.20 Hz, 2H, H-2', $\left.6^{\prime}\right), 8.03\left(\mathrm{~d}, J=8.51 \mathrm{~Hz}, 2 \mathrm{H}, \mathrm{H}-3 ', 5{ }^{\prime}\right) ;{ }^{13} \mathrm{C}$ NMR (125 MHz, DMSO-d 6 ) $\delta: 28.48$ (s, 2C, C-1, C-2), 50.31 (s, 2C, C-4, C-5), 79.37 (s, 2C, C-3, C-6), 126.87 (s, 2C, C-2', C-6'), 130.27 (s, 2C, C-3', C-5'), 133.71 (s, 1C, C-4'), 135.51 (s, 1C, C-1'), 167.83 (s, 1C, C-7'), 177.07 (s, 2C, C-7, C-8); HR-MS (ESI): $m / z$ calcd for $\mathrm{C}_{30} \mathrm{H}_{26} \mathrm{~N}_{2} \mathrm{O}_{10} 2 \mathrm{Na}\left([2 \mathrm{M}+\mathrm{Na}]^{+}\right)$597.1531, found 597.1535.

Data for 6-l: white solid; yield, 71\%; mp: $140-141{ }^{\circ} \mathrm{C}$; ${ }^{1} \mathrm{H}$ NMR (500 MHz, $\left.\mathrm{CDCl}_{3}\right) \delta$ : $1.63-1.69$ (m, 2H, H-1, 2, exo), 1.88-1.94 (m, 2H, H-1, 2, endo), 3.03 (s, 2H, H-4, 5), 4.99 (d, J=2.21 Hz, 2H, $\mathrm{H}-3,6), 7.10-7.19$ (m, 2H, H-3', 5'), 7.22-7.29 (m, 2H, H-2', 6'); $\left.{ }^{13} \mathrm{C} \mathrm{NMR} \mathrm{(125} \mathrm{MHz,} \mathrm{CDCl}_{3}\right) \delta: 28.66$ (s, 2C, C-1, C-2), 50.04 (s, 2C, C-4, C-5), 79.57 (s, 2C, C-3, C-6), 116.09 (s, 2C, C-3', C-5'), 127.75 (s, 1C, C-1'), 128.35 (s, 2C, C-2', C-6'), 163.29 (s, 1C, C-4'), 176.27 (s, 2C, C-7, C-8); HR-MS (ESI): $m / z$ calcd for $\mathrm{C}_{14} \mathrm{H}_{12} \mathrm{FNO}_{3} \mathrm{Na}\left([\mathrm{M}+\mathrm{Na}]^{+}\right)$284.0699, found 284.0675 .

Data for 6-m: white solid; yield, 85\%; mp: $177-178{ }^{\circ} \mathrm{C}$; ${ }^{1} \mathrm{H}$ NMR $\left(500 \mathrm{MHz}, \mathrm{CDCl}_{3}\right) \delta$ : 1.67 (d, $J=7.57 \mathrm{~Hz}, 2 \mathrm{H}, \mathrm{H}-1,2$, exo), 1.92 (d, $J=7.88 \mathrm{~Hz}, 2 \mathrm{H}, \mathrm{H}-1,2$, endo), 3.05 (s, 2H, H-4, 5), 5.00 (br, s, 2H, H-3, 6), 7.28-7.33 (m, 2H, H-3', 5'), 7.33-7.38 (m, 2H, H-2', 6'); ${ }^{13} \mathrm{C}$ NMR (125 MHz, $\left.\mathrm{CDCl}_{3}\right) \delta: 28.66$ (s, 2C, C-1, C-2) 50.05 (s, 2C, C-4, C-5) 79.62 (s, 2C, C-3. C-6) 119.36 (s, 1C, C-1') 121.60 (s, 2C, C-3', C-5') 127.98 (s, 2C, C-2', C-6') 130.22 (s, 1C, C-4') 148.89 (s, 1C, C-7') 176.07 (s, 2C, C-7, C-8); HR-MS (ESI): $m / z$ calcd for $\mathrm{C}_{15} \mathrm{H}_{12} \mathrm{~F}_{3} \mathrm{NO}_{4} \mathrm{Na}\left([\mathrm{M}+\mathrm{Na}]^{+}\right)$350.0616, found 350.0615 .

\subsubsection{General Synthetic Procedure for the Target Compounds 7 (a-m)}

A mixture of norcantharidin $3(1.0 \mathrm{~g}, 5.95 \mathrm{mmol})$, primary amine regent (1 equiv, $5.95 \mathrm{mmol})$ and THF $(10 \mathrm{~mL})$ was stirred for several hours at room temperature, and the progress of reaction was monitored by TLC. After norcantharidin disappeared, the reaction was concentrated under reduced pressure and diluted with acetone $(100 \mathrm{~mL})$. The resulting precipitate was either recrystallized from methanol or purified by column chromatography $\left(\mathrm{MeOH} / \mathrm{CH}_{2} \mathrm{Cl}_{2}, 1: 4, v / v\right)$ to afford the desired products 7. The yields, physical properties, ${ }^{1} \mathrm{H}$ NMR, ${ }^{13} \mathrm{C}$ NMR, and HR-MS of the target compounds 7 were as follows:

Data for 7-a: white solid; yield, 84\%; mp: $144-146{ }^{\circ} \mathrm{C} ;{ }^{1} \mathrm{H}$ NMR $\left(500 \mathrm{MHz}, \mathrm{D}_{2} \mathrm{O}\right) \delta: 1.45-1.70$ (m, 4H, H-1, 2), 2.57 (s, 3H, H-1'), 2.88-2.95 (m, 2H, H-4, 5), 4.60 (d, J=4.73 Hz, 1H, H-6), 4.79 $(\mathrm{d}, J=4.41 \mathrm{~Hz}, 1 \mathrm{H}, \mathrm{H}-3) ;{ }^{13} \mathrm{C}$ NMR $\left(125 \mathrm{MHz}, \mathrm{D}_{2} \mathrm{O}\right) \delta: 25.87(\mathrm{C}-1$ '), 28.20 (C-1, C-2), $53.84(\mathrm{C}-4)$, 54.70 (C-5), 78.68 (C-3), 79.39 (C-6), 175.58 (C-8), 177.81 (C-7); HR-MS (ESI): $\mathrm{m} / \mathrm{z}$ calcd for $\mathrm{C}_{9} \mathrm{H}_{12} \mathrm{NO}_{4}\left([\mathrm{M}-1]^{-}\right)$198.0766, found 198.0753.

Data for 7-b: white solid; yield 74\%; mp: 136-137 ${ }^{\circ} \mathrm{C}$; ${ }^{1} \mathrm{H}$ NMR (500 MHz, DMSO-d 6 ) $\delta: 1.02$ (dd, $\left.J=6.31,2.52 \mathrm{~Hz}, 6 \mathrm{H}, \mathrm{H}-2^{\prime}, 3^{\prime}\right), 1.41-1.62$ (m, 4H, H-1. 2), 2.75-2.85 (m, 2H, H-4, 5), 3.76 (dq, $\left.J=13.52,6.74 \mathrm{~Hz}, 1 \mathrm{H}, \mathrm{H}-1^{\prime}\right), 4.47$ (d, $\left.J=4.73 \mathrm{~Hz}, 1 \mathrm{H}, \mathrm{H}-6\right), 4.72$ (d, $J=4.10 \mathrm{~Hz}, 1 \mathrm{H}, \mathrm{H}-3$ ), 7.16 
(d, $J=7.88 \mathrm{~Hz}, 1 \mathrm{H}, \mathrm{H}-9) ;{ }^{13} \mathrm{C}$ NMR (125 MHz, DMSO-d 6 ) $\delta: 22.67$ (s, 1C, C-2'), 22.88 (s, 1C, C-3'), 28.93 (s, 1C, C-1), 29.31 (s, 1C, C-2), 43.13 (s, 1C, C-1'), 52.60 (s, 1C, C-3), 53.60 (s, 1C, C-4), 77.41 (s, 1C, C-3), 79.08 (s, 1C, C-6), 170.20 (s, 1C, C-8), 173.07 (s, 1C, C-7); HR-MS (ESI): $\mathrm{m} / \mathrm{z}$ calcd for $\mathrm{C}_{11} \mathrm{H}_{18} \mathrm{NO}_{4}\left([\mathrm{M}+1]^{+}\right)$228.1236, found 228.1233.

Data for 7-c: white solid; yield, 83.5\%; mp: 139-140 ${ }^{\circ} \mathrm{C} ;{ }^{1} \mathrm{H}$ NMR (500 MHz, DMSO-d 6 ) $\delta: 0.88$ (t, $\left.J=7.25 \mathrm{~Hz}, 3 \mathrm{H}, \mathrm{H}-4^{\prime}\right), 1.23-1.32$ (m, 2H, H-3'), 1.32-1.40 (m, 2H, H-2'), 1.42-1.63 (m, 4H, H-1, 2), 2.81-2.87 (m, 2H, H-1'), 2.94-3.05 (m, 2H, H-4, 5), 4.48 (d, J = 4.41 Hz, 1H, H-6), 4.74 (d, $J=4.10 \mathrm{~Hz}, 1 \mathrm{H}, \mathrm{H}-3), 7.33$ (t, $J=5.20 \mathrm{~Hz}, 1 \mathrm{H}, \mathrm{H}-9), 11.89$ (br, s, 1H, H-10); ${ }^{13} \mathrm{C}$ NMR (125 MHz,

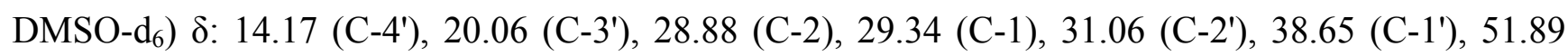
(C-4), 53.56 (C-5), 77.22 (C-3), 79.28 (C-6), 170.76 (C-8), 172.82 (C-7); HR-MS (ESI): $m / z$ calcd for $\mathrm{C}_{12} \mathrm{H}_{19} \mathrm{NO}_{4} \mathrm{Na}\left([\mathrm{M}+\mathrm{Na}]^{+}\right)$264.1212, found 264.1213.

Data for 7-d: white solid; yield, 66\%; Mp: $151-152{ }^{\circ} \mathrm{C} ;{ }^{1} \mathrm{H}$ NMR (500 MHz, DMSO-d 6 ) $\delta$ : 1.52-1.72 (m, 4H, H-1, 2), 3.05-3.17 (m, 2H, H-4, 5), 3.83 (s, 3H, H-7'), 4.71 (d, J = 5.04 Hz, 1H, H-6'), 4.89 (d, $J=3.47$ Hz, 1H, H-3'), 6.86-6.93 (m, 1H, H-5'), 7.02 (d, J=4.10 Hz, 2H, H-3', 4'), 8.11 (d, $J=7.88 \mathrm{~Hz}, 1 \mathrm{H}, \mathrm{H}-6$ '), 8.87 (s, 1H, H-9), 12.20 (br, s, 1H, H-10); ${ }^{13} \mathrm{C}$ NMR (125 MHz, DMSO-d 6 )

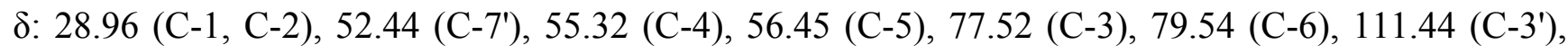
120.32 (C-6'), 120.79 (C-5'), 123.84 (C-3'), 128.31 (C-4'), 148.84 (C-2'), 169.95 (C-8), 172.61 (C-7); HR-MS (ESI): $m / z$ calcd for $\mathrm{C}_{15} \mathrm{H}_{17} \mathrm{NO}_{5} \mathrm{Na}\left([\mathrm{M}+\mathrm{Na}]^{+}\right) 314.1004$, found 314.1008 .

Data for 7-e: white solid; yield, 85\%; mp: $114-115{ }^{\circ} \mathrm{C}$; ${ }^{1} \mathrm{H}$ NMR (500 MHz, DMSO-d 6 ) $\delta$ : 1.53-1.68 (m, 4H, H-1, 2), 3.04 (d, $J=9.46 \mathrm{~Hz}, 1 \mathrm{H}, \mathrm{H}-4), 3.21$ (d, $J=9.77 \mathrm{~Hz}, 1 \mathrm{H}, \mathrm{H}-5$ ), 4.61-4.77 (m, 1H, H-6), 4.78-4.93 (m, 1H, H-3), 7.06-7.19 (m, 2H, H-3', 5'), 7.20-7.28 (m, 1H, H-4'), 8.02 $\left(\mathrm{t}, J=7.72 \mathrm{~Hz}, 1 \mathrm{H}, \mathrm{H}-6\right.$ '), 9.28 (s, 1H, H-9), 12.13 (br, s, 1H, H-10); ${ }^{13} \mathrm{C}$ NMR (125 MHz, DMSO-d 6 )

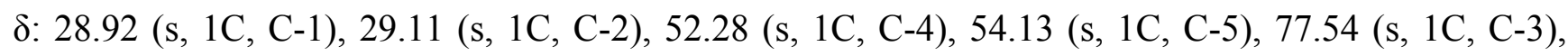
79.36 (s, 1C, C-6), 115.53 (s, 1C, C-3'), 123.24 (s, 1C, C-1'), 124.83 (s, 1C, 6'), 126.95 (s, 1C, 4'), 152.24 (s, 1C, C-5'), 154.17 (s, 1C, C-2'), 170.22 (s, 1C, C-8), 172.71 (s, 1C, C-7); HR-MS (ESI): $m / z$ calcd for $\mathrm{C}_{14} \mathrm{H}_{14} \mathrm{FNO}_{4} \mathrm{Na}\left([\mathrm{M}+\mathrm{Na}]^{+}\right)$302.0805, found 302.0807 .

Data for 7-f: yellow solid; yield, 47\%; mp: $160-161{ }^{\circ} \mathrm{C} ;{ }^{1} \mathrm{H}$ NMR (500 MHz, DMSO-d 6 ) $\delta$ : 1.46-1.72 (m, 4H, H-1, 2), 2.98-3.12 (m, 2H, H-4, 5), 4.63-4.85 (m, 2H, H-3, 6), 7.60 (t, J=8.20 Hz, 1H, H-4'), 7.82 (d, $\left.J=7.88 \mathrm{~Hz}, 1 \mathrm{H}, \mathrm{H}-5^{\prime}\right), 7.90$ (d, $J=7.88 \mathrm{~Hz}, 1 \mathrm{H}, \mathrm{H}-6$ '), 8.65 (br, s, 1H, H-3'), 10.28

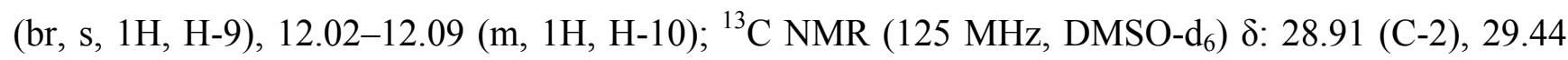
(C-1), 52.24 (C-4), 53.82 (C-5), 77.49 (C-3), 79.00 (C-6), 113.68 (C-6'), 117.96 (C-4'), 125.55 (C-3'), 130.53 (C-5'), 140.91 (C-1'), 148.42 (C-2'), 170.61 (C-8), 172.63 (C-7); HR-MS (ESI): $m / z$ calcd for $\mathrm{C}_{14} \mathrm{H}_{14} \mathrm{~N}_{2} \mathrm{O}_{6} \mathrm{Na} 329.0750$, found 329.0754.

Data for 7-g: white solid; yield, 73\%; mp: $165-166{ }^{\circ} \mathrm{C}$; ${ }^{1} \mathrm{H}$ NMR (500 MHz, DMSO-d 6 ) $\delta$ : 1.47-1.70 (m, 4H, H-1, 2), 2.95 (d, $J=9.46 \mathrm{~Hz}, 1 \mathrm{H}, \mathrm{H}-4), 3.07$ (d, $J=9.77 \mathrm{~Hz}, 1 \mathrm{H}, \mathrm{H}-5), 4.64$ (d, $J=4.41 \mathrm{~Hz}, 1 \mathrm{H}, \mathrm{H}-6), 4.80$ (d, $J=3.78 \mathrm{~Hz}, 1 \mathrm{H}, \mathrm{H}-3), 6.61(\mathrm{dd}, J=8.20,1.89 \mathrm{~Hz}, 1 \mathrm{H}, \mathrm{H}-4$ '), 7.05 (d, $\left.J=8.20 \mathrm{~Hz}, 1 \mathrm{H}, \mathrm{H}-6^{\prime}\right), 7.15-7.22$ (m, $\left.1 \mathrm{H}, \mathrm{H}-5^{\prime}\right), 7.29$ (s, 1H, H-2'), 9.68 (s, 1H, H-9), 11.99 (br, s, 1H, $\mathrm{H}-10) ;{ }^{13} \mathrm{C}$ NMR (125 MHz, DMSO-d 6 ) $\delta: 28.91$ (C-2), 29.46 (C-1), 52.05 (C-7'), 54.03 (C-4), 55.42 (C-5), 77.34 (C-3), 79.23 (C-6), 105.36 (C-6'), 108.97 (C-2'), 111.89 (C-4'), 129.81 (C-5'), 140.95 (C-1'), 159.95 (C-3'), 169.83 (C-8), 172.72 (C-7); HR-MS (ESI): $m / z$ calcd for $\mathrm{C}_{15} \mathrm{H}_{17} \mathrm{NO}_{5} \mathrm{Na}\left([\mathrm{M}+\mathrm{Na}]^{+}\right)$ 314.1004, found 314.0983 . 
Data for 7-h: white solid; yield, 86\%; mp: $163-164{ }^{\circ} \mathrm{C} ;{ }^{1} \mathrm{H}$ NMR (500 MHz, DMSO-d 6 ) $\delta$ : 1.41-1.71 (m, 4H, H-1, 2), 2.97 (d, $J=9.46 \mathrm{~Hz}, 1 \mathrm{H}, \mathrm{H}-4), 3.07$ (d, $J=9.46 \mathrm{~Hz}, 1 \mathrm{H}, \mathrm{H}-5), 4.66$ (d, $J=4.41 \mathrm{~Hz}, 1 \mathrm{H}, \mathrm{H}-6), 4.80$ (d, $J=3.78 \mathrm{~Hz}, 1 \mathrm{H}, \mathrm{H}-3), 6.85$ (td, $J=8.43,2.36 \mathrm{~Hz}, 1 \mathrm{H}, \mathrm{H}-4$ '), 7.22 $\left(\mathrm{d}, J=8.20 \mathrm{~Hz}, 1 \mathrm{H}, \mathrm{H}-6^{\prime}\right), 7.29-7.36\left(\mathrm{~m}, 1 \mathrm{H}, \mathrm{H}-5^{\prime}\right), 7.57$ (d, $\left.J=11.66 \mathrm{~Hz}, 1 \mathrm{H}, \mathrm{H}-2^{\prime}\right), 9.95$ (s, 1H, H-9) 12.01 (s, 1H, H-10); ${ }^{13} \mathrm{C}$ NMR (125 MHz, DMSO-d 6 ) $\delta: 28.89$ (s, 1C, C-1), 29.44 (s, 1C, C-2), 52.17 (s, 1C, C-4), 53.89 (s, 1C, C-5), 77.38 (s, 1C, C-3), 79.07 (s, 1C, C-6), 106.28 (s, 1C, C-4'), 109.72 (s, 1C, C-2'), 115.31 (s, 1C, C-6'), 130.62 (s, 1C, C-5'), 141.46 (s, 1C, C-1'), 161.65 (s, 1C, C-3'), 170.19 (s, 1C, C-8), 172.64 (s, 1C, C-7); HR-MS (ESI): $\mathrm{m} / \mathrm{z}$ calcd for $\mathrm{C}_{14} \mathrm{H}_{14} \mathrm{FNO}_{4} \mathrm{Na}\left([\mathrm{M}+\mathrm{Na}]^{+}\right)$302.0805, found 302.0811 .

Data for 7-i: white solid; yield, 49\%; mp: $174-176{ }^{\circ} \mathrm{C}$; ${ }^{1} \mathrm{H}$ NMR (500 MHz, DMSO-d 6 ) $\delta$ : 1.43-1.76 (m, 4H, H-1, 2), 2.99 (d, $J=9.46 \mathrm{~Hz}, 1 \mathrm{H}, \mathrm{H}-4), 3.09$ (d, $J=9.46 \mathrm{~Hz}, 1 \mathrm{H}, \mathrm{H}-5), 4.69$ (d, $J=2.84 \mathrm{~Hz}, 1 \mathrm{H}, \mathrm{H}-6), 4.80$ (br, s, 1H, H-3), 7.38 (d, $J=7.57 \mathrm{~Hz}, 1 \mathrm{H}, \mathrm{H}-5$ ') 7.54 (t, $J=7.88 \mathrm{~Hz}$, 1H, H-4'), 7.68 (d, J = 7.88 Hz, 1H, H-6'), 8.12 (br, s, 1H, H-2'), 10.11 (s, 1H, H-9), 12.04 (s, 1H, $\mathrm{H}-10) ;{ }^{13} \mathrm{C}$ NMR (125 MHz, DMSO-d 6 ) $\delta: 28.90$ (s, 1C, C-1), 29.43 (s, 1C, C-2), 52.19 (s, 1C, C-4), 53.85 (s, 1C, C-5), 77.43 (s, 1C, C-3), 79.04 (s, 1C, C-6), 115.67 (s, 1C, C-4'), 119.70 (s, 1C, C-7'), 123.08 (s, 1C, C-6'), 123.56 (s, 1C, C-2'), 125.72 (s, 1C, C-5'), 130.29 (s, 1C, C-3'), 140.51 (s, 1C, C-1'), 170.43 (s, 1C, C-8), 172.67 (s, 1C, C-7); HR-MS (ESI): $m / z$ calcd for $\mathrm{C}_{15} \mathrm{H}_{14} \mathrm{~F}_{3} \mathrm{NO}_{4} \mathrm{Na}$ $\left([\mathrm{M}+\mathrm{Na}]^{+}\right)$352.0773, found 352.0775.

Data for 7-j: white solid; yield, 71\%; mp: $167-168{ }^{\circ} \mathrm{C}$; ${ }^{1} \mathrm{H}$ NMR (500 MHz, DMSO-d 6 ) $\delta$ : 1.46-1.69 (m, 4H, H-1, 2), 2.94 (d, $J=9.77 \mathrm{~Hz}, 1 \mathrm{H}, \mathrm{H}-4), 3.03$ (d, $J=9.46 \mathrm{~Hz}, 1 \mathrm{H}, \mathrm{H}-5), 4.63$ (d, $J=4.10 \mathrm{~Hz}, 1 \mathrm{H}, 1 \mathrm{H}, \mathrm{H}-6), 4.79$ (d, $J=3.78 \mathrm{~Hz}, 1 \mathrm{H}, 1 \mathrm{H}, \mathrm{H}-3), 6.87$ (d, $\left.J=8.83 \mathrm{~Hz}, 2 \mathrm{H}, \mathrm{H}-3^{\prime}, 5^{\prime}\right)$, 7.44 (d, $J=8.83 \mathrm{~Hz}, 2 \mathrm{H}, \mathrm{H}-2$ ', 6'), 9.51 (s, 1H, H-9), 11.93 (br, s, 1H, H-10); ${ }^{13} \mathrm{C}$ NMR (125 MHz, DMSO-d 6 ) $\delta: 28.89$ (C-2), 29.47 (C-1), 52.07 (C-7'), 53.87 (C-4), 55.64 (C-5), 77.29 (C-3), 79.20 (C-6), 114.20 (C-3', C-5'), 121.24 (C-2', C-6'), 132.91 (C-1'), 155.54 (C-4'), 169.33 (C-8), 172.76 (C-7); HR-MS (ESI): $m / z$ calcd for $\mathrm{C}_{15} \mathrm{H}_{17} \mathrm{NO}_{5} \mathrm{Na}\left([\mathrm{M}+\mathrm{Na}]^{+}\right)$314.1004, found 314.1025.

Data for 7-k: white solid; yield, 44\%; mp: 269-270 ${ }^{\circ} \mathrm{C}$; ${ }^{1} \mathrm{H}$ NMR (500 MHz, DMSO-d 6 ) $\delta$ : 1.52-1.64 (m, 4H, H-1, 2), 2.98 (d, $J=9.46 \mathrm{~Hz}, 1 \mathrm{H}, \mathrm{H}-4), 3.11$ (d, $J=9.77 \mathrm{~Hz}, 1 \mathrm{H}, \mathrm{H}-5), 4.68$ (d, $J=4.41 \mathrm{~Hz}, 1 \mathrm{H}, \mathrm{H}-6), 4.80$ (d, $J=3.78 \mathrm{~Hz}, 1 \mathrm{H}, \mathrm{H}-3), 7.66$ (d, $J=8.83 \mathrm{~Hz}, 2 \mathrm{H}, \mathrm{H}-2$ ', 6 '), 7.89 (d, $J=8.83 \mathrm{~Hz}, 2 \mathrm{H}, \mathrm{H}-3$ ', 5'), 10.05 (s, 1H, H-9), 12.35 (br, s, 2H, H-10); ${ }^{13} \mathrm{C}$ NMR (125 MHz, $\left.\mathrm{DMSO}_{-} \mathrm{d}_{6}\right) \delta: 28.91$ (s, 1C, C-1) 29.44 (s, 1C, C-2) 52.20 (s, 1C, C-4) 53.97 (s, 1C, C-5) 77.42 (s, 1C, C-3) 79.11 (s, 1C, C-6) 118.80 (s, 2C, C-2', C-6') 125.29 (s, 1C, C-4') 130.78 (s, 2C, C-3', C-5') 143.69 (m, 1C, C-1') 167.32 (m, 1C, C-7') 170.32 (s, 1C, C-8) 172.65 (s, 1C, C-7); HR-MS (ESI): m/z calcd for $\mathrm{C}_{15} \mathrm{H}_{15} \mathrm{NO}_{6} \mathrm{Na}\left([\mathrm{M}+\mathrm{Na}]^{+}\right)$328.0797, found 328.0796.

Data for 7-l: white solid; yield, 78\%; mp: $157-159{ }^{\circ} \mathrm{C}$; ${ }^{1} \mathrm{H}$ NMR (500 MHz, DMSO-d 6 ) $\delta$ : 1.45-1.69 (m, 4H, H-1, 2), 2.93-2.98 (m, 1H, H-4), 3.02-3.08 (m, 1H, H-5), 4.65 (d, J=4.10 Hz, 1H, H-6), 4.79 (d, $J=3.78 \mathrm{~Hz}, 1 \mathrm{H}, \mathrm{H}-3), 7.13$ (t, $\left.J=8.83 \mathrm{~Hz}, 2 \mathrm{H}, \mathrm{H}-2^{\prime}, 6^{\prime}\right), 7.55$ (dd, $J=8.83,5.04 \mathrm{~Hz}$, 2H, H-3', 5'), 9.73 (s, 1H, H-8), 11.98 (br, s, 1H, H-7); ${ }^{13} \mathrm{C}$ NMR (125 MHz, DMSO-d 6 ) $\delta: 28.90$ (s, 1C, C-1), 29.46 (s, 1C, C-2), 52.15 (s, 1C, C-4), 53.83 (s, 1C, C-5), 77.34 (s, 1C, C-3), 79.10 (s, 2C, C-6), 115.49 (s, 2C, C-3', C-5'), 121.37 (s, 2C, C-2', C-6'), 136.13 (s, 1C, C-1'), 157.37 (s, 1C, C-4'), 169.71 (s, 1C, C-8), 172.68 (s, 1C, C-7); HR-MS (ESI): $m / z$ calcd for $\mathrm{C}_{14} \mathrm{H}_{14} \mathrm{FNO}_{4} \mathrm{Na}\left([\mathrm{M}+\mathrm{Na}]^{+}\right)$ 302.0805 , found 302.0813 . 
Data for 7-m: yellow solid; yield, 47\%; mp: 170-171 ${ }^{\circ} \mathrm{C} ;{ }^{1} \mathrm{H}$ NMR (500 MHz, DMSO-d 6 ) $\delta$ : 1.46-1.69 (m, 4H, H-1, 2), 2.95-3.00 (m, 1H, H-4), 3.07 (d, $J=9.77 \mathrm{~Hz}, 1 \mathrm{H}, \mathrm{H}-5), 4.67$ (d, $J=4.41 \mathrm{~Hz}, 1 \mathrm{H}, \mathrm{H}-6), 4.80$ (d, $J=3.78 \mathrm{~Hz}, 1 \mathrm{H}, \mathrm{H}-3), 7.30$ (d, $\left.J=8.83 \mathrm{~Hz}, 2 \mathrm{H}, \mathrm{H}-3^{\prime}, 5^{\prime}\right), 7.65$ (d, $\left.J=8.83 \mathrm{~Hz}, 2 \mathrm{H}, \mathrm{H}-2^{\prime}, 6^{\prime}\right), 9.91$ (s, 1H, H-9), 12.01 (s, 1H, H-10); ${ }^{13} \mathrm{C}$ NMR (125 MHz, DMSO-d 6 )

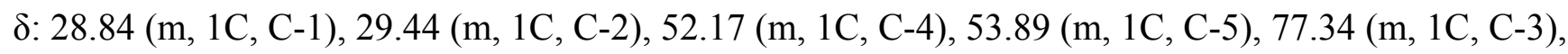
79.07 (m, 1C, C-6), 120.88 (s, 2C, C-3', C-5'), 121.65 (s, 1C, C-7'), 121.97 (s, 2C, C-2', C-6'), 138.91 (m, 1C, C-1'), 143.80 (m, 1C, C-4'), 169.85 (m, 1C, C-8), 172.47 (m, 1C, C-7); HR-MS (ESI): $m / z$ calcd for $\mathrm{C}_{15} \mathrm{H}_{14} \mathrm{~F}_{3} \mathrm{NO}_{5} \mathrm{Na}\left([\mathrm{M}+\mathrm{Na}]^{+}\right)$368.0722, found 368.0732 .

\subsection{Bioassay}

Plutella xylostella were continuously maintained in our laboratory without exposure to any insecticide at $25 \pm 2{ }^{\circ} \mathrm{C}, 50 \% \mathrm{RH}$ with a photoperiod of $16 \mathrm{~L}: 8 \mathrm{D}$. The larvae were reared on pakchoi seedlings.

Compounds were dissolved in component solvent (water:acetone:dimethyl sulfoxide $=20: 19: 1, v / v$ ) containing $0.5 \%$ Tween- 80 to the different concentrations. The bioactivities of all compounds against the early third instar larvae of $P$. xylostella were tested using cotyledons of pakchoi seedlings. The seedlings were treated by firstly dipping them in the test solutions for about $5 \mathrm{~s}$, then placing cotyledons slanting over a blotting paper to drain superfluous fluid and to dry the test solution for about $25 \mathrm{~min}$ at room temperature.

For each bioassay, ten treated leaves were put on a wet filter paper $(\varnothing 9 \mathrm{~cm})$ paved in a Petri plate $(\varnothing 9 \mathrm{~cm})$ to keep fresh, and ten larvae were released to the plate after a starvation for $4 \mathrm{~h}$, and kept in a growth cabinet under the same conditions the larvae were reared. Each sample was repeated four times and mortality was recorded after $48 \mathrm{~h}$. A component solvent containing $0.5 \%$ Tween- 80 was used as control. Bioassay data were polled and analyzed by standard probit analysis [23], using Abbott's correction for control mortality [24], and the toxicity was ascertained by estimating the median lethal dose $\left(\mathrm{LD}_{50}\right.$, killing $\left.50 \%\right)$ of each compound.

\section{Conclusions}

In summary, two series of cantharidin analogs (compounds 6 and compounds 7) containing alkyl and aryl groups at 9-position were designed and synthesized. Their structures were confirmed by ${ }^{1} \mathrm{H}$ NMR, ${ }^{13} \mathrm{C}$ NMR and HRMS. The larvicidal activities of cantharidin and its derivatives against the pre-third-instar P. xylostella were evaluated. All of the cyclic compounds except cantharidin $\mathbf{1}$ and norcantharidin $\mathbf{3}$ lacked any larvicidal activity. The ring-opened cantharidin derivatives demonstrated different activities, and compound $7 \mathbf{f}$ showed the highest larvicidal activity with $\mathrm{LC}_{50}$ value of $0.43 \mathrm{mM}$. Structure-activity relationship study indicated that the form of compound (cyclic or ring-opened) or their ability to hydrolyze facilely is the key to determine whether it exhibits larvicidal activity. Meanwhile, this revealed that the improvement of insecticidal activity required a reasonable combination of both aliphatic amide and aromatic amide moieties, and the type of substituent $\mathrm{Y}$ on the aniline ring was critical. 


\section{Acknowledgments}

We appreciate the kind help and helpful advice given by Xuan Tian (Lanzhou University, China) and Hong-Li Zhang (Northwest A \& F University, China) during the spectroscopic analysis on the anhydride modified derivatives of Cantharidin. We also give thanks to J. R. Schrock (Emporia State University, USA) for revising the manuscript. This study was supported by the Special Fund for the Public Interest (Agriculture) (Grant No. 200903052) and the "13115" Sci-Tech Innovation Project of Shaanxi Province (No. 2009ZDKG-06).

\section{References}

1. Kuo, J.H.; Chu, Y.L.; Yang, J.S.; Lin, J.P.; Lai, K.C.; Kuo, H.M.; Hsia, T.C.; Chung, J.G. Cantharidin induces apoptosis in human bladder cancer TSGH 8301 cells through mitochondria-dependent signal pathways. Int. J. Oncol. 2010, 37, 1243-1250.

2. Huang, W.W.; Ko, S.W.; Tsai, H.Y.; Chung, J.G.; Chiang, J.H.; Chen, K.T.; Chen, Y.C.; Chen, Y.F.; Yang, J.S. Cantharidin induces G2/M phase arrest and apoptosis in human colorectal cancer colo 205 cells through inhibition of CDK1 activity and caspase-dependent signaling pathways. Int. J. Oncol. 2011, 38, 1067-1073.

3. Morihiko, S.; Tomonori, N.; Hideo, U.; Yasuo, I.; Masahiro, K. Cantharidin induces apoptosis of human multiple myeloma cells via inhibition of the JAK/STAT pathway. Cancer Sci. 2008, 99, $1820-1826$.

4. Chen, L.P.; Yang, B.D.; Zhang, Z.Y.; Wang, J.Z.; Sun, S.L. Effects of cantharidin on insect cell Spex-VII and Sf9. J. Beijing Univ. Agric. 2008, 23, 17-20.

5. Carrel, J.E.; Eisner, T. Cantharidin: Potent feeding deterrent to insects. Science 1974, 183, 755-757.

6. Li, X.F.; Chen, X.S.; Hou, X.H. The toxicity effect of cantharidin on six pests. Guizhou Agric. Sci. 2008, 36, 65-66.

7. Liu, R.R.; Ma, Y.; Ma, Z.Q.; Zhang, Y.L. Bioactivity of cantharidin against eleven pests. J. Northwest AF Univ. 2010, 38, 181-185.

8. Lu, B.L. Pathological changes following the administration of cantharidin to Leucania separata (Walker). Acta Entomol. Sin. 1964, 13, 824-834.

9. Zhang, Y.L.; Zhou, Y.; Zhang, Z.Y. Effect of cantharidin on the midgut of the orient armyworm (Mythimna separata) and diamondback moth (Plutella xylostella). Acta Entomol. Sin. 2003, 46, 272-276.

10. Chen, L.P.; Yang, B.D.; Zhang, Z.Y.; Zhang, A.H.; Zhang, Z.M.; Zhang, L.X.; Sun, S.L. Effect of cantharidin on the integument structure of Plutella xylostella. Entomol. Knowl. 2011, 48, 1779-1785.

11. Ma, Y.; Liu, R.R.; Ma, Z.Q.; Zhang, Y.L. Effects of cantharidin on four metabolizing enzymes and PPO in Mythimna separata (Walker) (Lepidoptera Noctuidae). Acta Entomol. Sin. 2010, 53, 870-875.

12. Srork, G.; Tamelen, E.; Friedman, L.J.; Burgstahler, A.W. Cantharidin. A stereospecific total synthesis. J. Am. Chem. Soc. 1951, 73, 4501.

13. Srork, G.; Tamelen, E.; Friedman, L.J.; Burgstahler, A.W. Stereospecific synthesis of cantharidin. J. Am. Chem. Soc. 1953, 75, 384-392. 
14. Dauben, W.G.; Kessel, C.R.; Takemura, K.H. Simple, efficient total synthesis of cantharidin via a high-pressure Diels-Alder reaction. J. Am. Chem. Soc. 1980, 102, 6893-6894.

15. Hill, T.A.; Stewart, S.G.; Ackland, S.P.; Gilbert, J.; Sauer, B.; Sakoff, J.A.; McCluskey, A. Norcantharimides, synthesis and anticancer activity: Synthesis of new norcantharidin analogues and their anticancer evaluation. Bioorg. Med. Chem. 2007, 15, 6126-6134.

16. Hill, T.A.; Stewart, S.G.; Gordon, C.P.; Ackland, S.P.; Gilbert, J.; Sauer, B.; Sakoff, J.A.; McCluskey, A. Norcantharidin analogues: Synthesis, anticancer activity and protein phosphatase 1 and 2A inhibition. ChemMedChem 2008, 3, 1878-1892.

17. Yue, H.Y.; Liu, Z.F. A new synthetic process of phthalimide. Fine Chem. Inter. 2004, 35, 44-45.

18. Bajsa, J.; Duke, S.O.; Tekwani, B.L. Plasmodium falciparum serine/threonine phosphoprotein phosphatases (PPP): From housekeeper to the "Holy Grail". Curr. Drug Targets 2008, 9, 997-1012.

19. Lin, X.H.; Walter, J.; Scheidmann, K.; Ohst, K.; Newport, J.; Walter, G. Protein phosphatase 2A is required for the initiation of chromosomal DNA replication. Proc. Natl. Acad. Sci. USA 1998, 95, 14693-14698.

20. Moorhead, G.B.; de Wever, V.; Templeton, G.; Kerk, D. Evolution of protein phosphatases in plants and animals. Biochem. J. 2009, 417, 401-409.

21. Bertini, I.; Calderone, V.; Fragai, M.; Luchinat, C.; Talluri, E. Structural basis of serine/threonine phosphatase inhibition by the archetypal small molecules cantharidin and norcantharidin. J. Med. Chem. 2009, 52, 4838-4843.

22. Li, Y.M.; Mackintosh, C.; Casida J.E. Protein phosphatase $2 \mathrm{~A}$ and its $\left[{ }^{3} \mathrm{H}\right]$ cantharidin $/\left[{ }^{3} \mathrm{H}\right]$ endothall thioanhydride binding site. Inhibitor specificity of cantharidin and ATP analogues. Biochem. Pharmacol. 1993, 46, 1435-1443.

23. Finney, D.J. Probit Analysis, 2nd ed.; Cambridge University Press: Cambridge, UK, 1971; pp. $35-41$.

24. Abbott, W.S. A method of computing the effectiveness of an insecticide. J. Econ. Entomol. 1925, 18, 265-267.

(C) 2013 by the authors; licensee MDPI, Basel, Switzerland. This article is an open access article distributed under the terms and conditions of the Creative Commons Attribution license (http://creativecommons.org/licenses/by/3.0/). 